\title{
Relativistic four-component calculations of Buckingham birefringence using London atomic orbitals
}

\author{
Radovan Bast • Kenneth Ruud • Antonio Rizzo • \\ Trygve Helgaker
}

Received: 1 January 2011/Accepted: 5 April 2011 / Published online: 28 April 2011

(C) The Author(s) 2011. This article is published with open access at Springerlink.com

\begin{abstract}
We present the first relativistic study of the electric-field-gradient induced birefringence (Buckingham birefringence), with application to the series of molecules $\mathrm{CX}_{2}(\mathrm{X}=\mathrm{O}, \mathrm{S}, \mathrm{Se}, \mathrm{Te})$. A recently developed atomicorbital-driven scheme for the calculation of time-dependent molecular properties using one-, two- and four-component relativistic wave functions (Bast et al. in Chem Phys $356: 177$, 2009) is extended to first-order frequencydependent magnetic-field perturbations, using London atomic orbitals to ensure gauge-origin independent results and to improve basis-set convergence. Calculations are presented at the Hartree-Fock and Kohn-Sham levels of theory and results for $\mathrm{CO}_{2}$ and $\mathrm{CS}_{2}$ are compared with previous high-level coupled-cluster calculations. Except
\end{abstract}

Dedicated to Professor Pekka Pyykkö on the occasion of his 70th birthday and published as part of the Pyykkö Festschrift Issue.

\section{R. Bast $(\bowtie) \cdot K$. Ruud}

Department of Chemistry, Centre for Theoretical and

Computational Chemistry (CTCC), University of Troms $\varnothing$,

9037 Troms $\varnothing$, Norway

e-mail: radovan.bast@uit.no

K. Ruud

e-mail: kenneth.ruud@uit.no

\footnotetext{
A. Rizzo

CNR - Consiglio Nazionale delle Ricerche,

Istituto per i Processi Chimico Fisici (IPCF-CNR),

UoS di Pisa, Area della Ricerca, via G. Moruzzi 1,

56124 Pisa, Italy

e-mail: rizzo@ipcf.cnr.it

T. Helgaker

Department of Chemistry, Centre for Theoretical

and Computational Chemistry (CTCC), University of Oslo,

P.O. Box 1033, Blindern, 0315 Oslo, Norway

e-mail: trygve.helgaker@kjemi.uio.no
}

for the heaviest member of the series, relativistic effects are small-in particular for the temperature-independent contribution to the birefringence. By contrast, the effects of electron correlation are significant. However, the reliability of standard exchange-correlation functionals in describing Buckingham birefringence remains unclear based on the comparison with high-level coupled-cluster singles-anddoubles calculations.

Keywords Relativistic effects · Buckingham birefringence $\cdot$ Nonlinear properties $\cdot$ Response theory

\section{Introduction}

More than half a century ago, Buckingham [1, 2] proposed that measurements of the birefringence induced by a gradient of an external electric field on linearly polarized radiation passing through a sample of molecules could be used as a direct method to measure molecular quadrupole moments [3]. Today, this idea has evolved into an important technique for determining the molecular quadrupole moment, which is the leading electric multipole in nonpolar molecules and as such plays a major role in determining the structural and spectroscopic behavior of matter [4-7].

The electric-field-gradient induced (linear) birefringence, also known as the Buckingham effect or Buckingham birefringence, is measured by the optical retardation, proportional to the anisotropy $n_{x}-n_{y}$, generated in the real part of the complex refractive index when linearly polarized radiation travels in the $z$ direction through a fluid immersed in an electric field gradient with components in the $x y$ plane. The phase difference is directly proportional to the optical path length and inversely proportional to the wave length of the impinging radiation. As a result of the 
interactions between the radiation field, the electric field gradient and the sample, the beam exiting the sample cell is elliptically polarized.

To lowest order in a perturbation expansion involving fields and molecular multipoles at constant pressure, Buckingham birefringence has two contributions. The first accounts for the thermal orientational effect of the electric field gradient on the freely rotating molecules and is inversely proportional to the temperature for a measurement carried out at constant pressure. This contribution depends on the electric quadrupole moment and the electric-dipole polarizability tensors; for polar molecules, the mixed electric-dipole-electric-quadrupole and electric-dipole-magnetic-dipole polarizabilities contribute in addition to the static molecular dipole moment [4]. The second, temperature-independent contribution to Buckingham birefringence arises from the response of the molecular electronic system to the external field and depends on higher-order mixed response properties. Although typically smaller than the temperature-dependent contribution, it is the only non-vanishing contribution to Buckingham birefringence in fluids consisting of atoms and spherically symmetric molecules.

In recent years, we have contributed to the study of Buckingham birefringence by developing and applying computational techniques for the $a b$ initio prediction of the effect-see Refs. [8-11]. Since our first investigation of Buckingham birefringence in 1998 [12], which focused on the nonpolar molecules $\mathrm{H}_{2}, \mathrm{~N}_{2}, \mathrm{C}_{2} \mathrm{H}_{2}$ and $\mathrm{CH}_{4}$ and led to a revised experimental quadrupole moment of $\mathrm{N}_{2}$ [13], we have extended our interest to larger and more complex systems, including polar [14, 15] as well as nonpolar [16-22] molecules, for which the effect of electron correlation and the choice of electronic-structure model have been analyzed in detail. In Ref. [23], the Buckingham birefringence of solvated molecules was investigated, thereby assessing the ability of our approach to simulate the effect of the environment. Our studies of carbon monoxide, nitrous oxide and carbonyl sulfide $[14,15]$ have resolved a controversy regarding the semiclassical theory of Buckingham birefringence [4, 24], supporting [25-27] the original derivation by Buckingham and Longuet-Higgins [4]. The dependence of Buckingham birefringence on the density for helium, neon and argon was analyzed in Ref. [28]. Most recently, we presented an approach to the study of Buckingham birefringence with perturbation-dependent basis sets that ensures invariance of the results to the origin of the multipole expansion [22].

An unexplored aspect of Buckingham birefringence is the influence of relativity. In this communication, we study relativistic effects on the Buckingham birefringence for the series of molecules $\mathrm{CX}_{2}(\mathrm{X}=\mathrm{O}, \mathrm{S}, \mathrm{Se}, \mathrm{Te})$. For $\mathrm{CO}_{2}, \mathrm{CS}_{2}$ and $\mathrm{CSe}_{2}$, we compare our results with previous nonrelativistic ab initio [17] data and with experiment [29-34]. The atomic-orbital-driven (AO-driven) scheme recently introduced by Bast et al. [35] for calculating time-dependent molecular properties with one-, two- and four-component relativistic methods is extended to first-order frequency-dependent magnetic perturbations with London atomic orbitals (LAOs) [36-40], thereby ensuring gaugeorigin independence of the calculated results. The present work can also be considered an extension of our recent analytic implementation of Buckingham birefringence [22] to Kohn-Sham (KS) density-functional theory (DFT). Results are presented at the Hartree-Fock (HF) and KS levels of theory using nonrelativistic and four-component relativistic (reference) wave functions.

\section{Theory}

We here review the theory underlying the Buckinghambirefringence calculations presented in this paper. First, the important quantities of Buckingham birefringence are reviewed in Sect. 2.1. Following a discussion of our treatment of relativity in Sect. 2.2, we outline the response theory used to calculate Buckingham birefringence at the relativistic HF and KS levels of theory in Sect. 2.3.

\subsection{Buckingham birefringence}

For an ideal gas at constant pressure, the birefringence induced by applying an electric field gradient

$\nabla E=\nabla E_{X X}=-\nabla E_{Y Y}, \quad \nabla E_{Z Z}=0$,

to a beam propagating in the $z$ direction with circular frequency $\omega$ is given by the expression $[3,4,6,25,41]$

$\Delta n=n_{X}-n_{Y}=\frac{3 \nabla E}{2 V_{\mathrm{m}}}{ }_{\mathrm{m}} Q(\omega, T)$,

where $V_{\mathrm{m}}$ is the molar volume and ${ }_{\mathrm{m}} Q(\omega, T)$ is the Buckingham constant. For nonpolar systems, for which the molecular quadrupole moment does not depend on the choice of origin $[1,25,41]$, the Buckingham constant (in the Einstein summation convention),

${ }_{\mathrm{m}} Q(\omega, T)=\frac{N_{\mathrm{A}}}{3 \varepsilon_{0}}\left[b(\omega)+\frac{2}{15 k T} \Theta_{\alpha \beta} \alpha_{\alpha \beta}(-\omega ; \omega)\right]$,

consists of a temperature-independent term $b(\omega)$ and a temperature-dependent term bilinear in the quadrupolemoment components $\Theta_{\alpha \beta}$ and in the frequency-dependent electric-dipole polarizability components, obtained from linear response theory as

$\alpha_{\alpha \beta}(-\omega ; \omega)=-\left\langle\left\langle\hat{\mu}_{\alpha} ; \hat{\mu}_{\beta}\right\rangle\right\rangle_{\omega}$.

In Eq. 3 above, $N_{\mathrm{A}}, k$, and $\varepsilon_{0}$ are the Avogadro, Boltzmann, and electric constants, respectively. The temperatureindependent term $b(\omega)$ in Eq. 3 is given as 


$$
\begin{aligned}
b(\omega)= & \frac{2}{15} B_{\alpha \beta, \alpha \beta}(-\omega ; \omega, 0)-\frac{2}{15} \mathscr{B}_{\alpha, \alpha \beta, \beta}(-\omega ; \omega, 0) \\
& -\frac{2}{3 \omega} \varepsilon_{\alpha \beta \gamma} J_{\alpha, \beta, \gamma}^{\prime}(-\omega ; \omega, 0),
\end{aligned}
$$

where $\varepsilon_{\alpha \beta \gamma}$ is the Levi-Civita symbol and where we have introduced the mixed polarizabilities

$$
\begin{aligned}
& B_{\alpha \beta, \gamma \delta}(-\omega ; \omega, 0)=B_{\alpha \beta, \gamma \delta}(\omega)=\left\langle\left\langle\hat{\mu}_{\alpha} ; \hat{\mu}_{\beta}, \hat{\Theta}_{\gamma \delta}\right\rangle\right\rangle_{\omega, 0}, \\
& \mathscr{B}_{\alpha, \beta \gamma, \delta}(-\omega ; \omega, 0)=\mathscr{B}_{\alpha, \beta \gamma, \delta}(\omega)=\left\langle\left\langle\hat{\mu}_{\alpha} ; \hat{\Theta}_{\beta \gamma}, \hat{\mu}_{\delta}\right\rangle\right\rangle_{\omega, 0} \\
& =\left\langle\left\langle\hat{\mu}_{\alpha} ; \hat{\mu}_{\delta}, \hat{\Theta}_{\beta \gamma}\right\rangle\right\rangle_{0, \omega}, \\
& J_{\alpha, \beta, \gamma}^{\prime}(-\omega ; \omega, 0)=J_{\alpha, \beta, \gamma}^{\prime}(\omega)=\mathrm{i}\left\langle\left\langle\hat{\mu}_{\alpha} ; \hat{m}_{\beta}, \hat{\mu}_{\gamma}\right\rangle\right\rangle_{\omega, 0} .
\end{aligned}
$$

In the equations above, the electric-dipole $\hat{\mu}_{\alpha}$, electricquadrupole $\hat{\Theta}_{\alpha \beta}$ and magnetic-dipole $\hat{m}_{\alpha}$ operators are given by (atomic units)

$$
\begin{aligned}
\hat{\mu}_{\alpha}= & \sum_{K} Z_{K} R_{K, \alpha}-\sum_{i} r_{i, \alpha}, \\
\hat{\Theta}_{\alpha \beta}= & \frac{1}{2} \sum_{K} Z_{K}\left(R_{K, \alpha} R_{K, \beta}-\frac{1}{3} \delta_{\alpha \beta} R_{K, \gamma} R_{K, \gamma}\right) \\
& -\frac{1}{2} \sum_{i}\left(r_{i, \alpha} r_{i, \beta}-\frac{1}{3} \delta_{\alpha \beta} r_{i, \gamma} r_{i, \gamma}\right), \\
\hat{m}_{\alpha}= & -\frac{c_{0}}{2} \sum_{i}\left(\mathbf{r}_{i} \times \alpha\right)_{\alpha},
\end{aligned}
$$

where the summations are over nuclei $K$ of charge $Z_{K}$ and positions $\mathbf{R}_{K}$ and over electrons $i$ of positions $\mathbf{r}_{i}$. In Eq. 8, $c_{0}$ is the speed of light in vacuum and $\alpha$ is the four-by-four matrix

$\boldsymbol{\alpha}=\left[\begin{array}{cc}0_{2 \times 2} & \boldsymbol{\sigma} \\ \boldsymbol{\sigma} & 0_{2 \times 2}\end{array}\right]$,

where $\sigma$ is the vector of two-by-two Pauli spin matrices in the standard representation [42]. We have not included the nuclear part of the magnetic-dipole operator, which does not contribute to Buckingham birefringence in the BornOppenheimer approximation.

As shown by Buckingham and Longuet-Higgins [4], for polar fluids, where the quadrupole moment is origin dependent, Eq. 3 must be generalized to include a correction to the temperature-dependent part involving the molecular dipole moment and the mixed electric-dipole-magneticdipole and electric-dipole-electric-quadrupole polarizabilities. As we only study nonpolar molecules in this work, we do not consider this generalization here, referring the interested reader to Refs. [4, 14, 22] for more information.

\subsection{Relativistic Hamiltonians and self-consistent-field wave functions}

Relativistic effects are the differences between the results of a relativistic calculation with $c_{0} \approx 137$ a.u. at a given level of electronic-structure theory and the corresponding results obtained at the same level of theory with $c_{0} \rightarrow \infty$. They can be studied perturbatively by adding relativistic corrections to a nonrelativistic description [43, 44], or nonperturbatively, by starting from a relativistic Hamiltonian with spin-orbit coupling included variationally and selectively removing relativistic contributions $[45,46]$. The latter approach is pursued in this work. Our point of reference is the four-component relativistic Hamiltonian which, in the Born-Oppenheimer approximation, may be written as a sum of one- and two-electron terms plus the classical nuclear electrostatic-repulsion term [47, 48]

$\hat{H}=\sum_{i} \hat{h}_{\mathrm{D}}(i)+\frac{1}{2} \sum_{i \neq j} \hat{g}(i, j)+V^{n n}$.

The one-electron Dirac operator $\hat{h}_{\mathrm{D}}$ is given by

$\hat{h}_{\mathrm{D}}=\left[\begin{array}{cc}V & c_{0} \boldsymbol{\sigma} \cdot \boldsymbol{\pi} \\ c_{0} \boldsymbol{\sigma} \cdot \boldsymbol{\pi} & -2 c_{0}^{2}+V\end{array}\right]$,

where $\pi=-\mathrm{i} \nabla+e \mathbf{A}$ is the kinetic momentum of an electron in the vector potential $\mathbf{A}$ and $V$ represents the scalar potential generated by the nuclei and the applied electromagnetic field. A closed Lorentz-invariant expression for the two-electron interaction does not exist and $\hat{g}(i, j)$ is to zeroth order represented by the instantaneous Coulomb interaction

$\hat{g}^{\mathrm{C}}(i, j)=\left(I_{4 \times 4} \otimes I_{4 \times 4}\right) r_{i j}^{-1}$.

In the resulting Dirac-Coulomb (DC) Hamiltonian, the Gaunt interaction [49] and higher-order relativistic electron-electron corrections are neglected [50-52]. In terms of lowest-order perturbation theory, this means that the mass-velocity, Darwin and spin-orbit effects are accounted for, while the spin-otherorbit, orbit-orbit and spin-spin effects are neglected.

The many-electron wave function is a linear combination of antisymmetrized products of complex four-component spinors

$\psi=\left[\begin{array}{l}\psi^{\mathrm{L} \alpha} \\ \psi^{\mathrm{L} \beta} \\ \psi^{\mathrm{S} \alpha} \\ \psi^{\mathrm{s} \beta}\end{array}\right]$,

consisting of large (L) and small (S) component bispinors. Relativistic effects can now be removed by increasing the speed of light in vacuum $c_{0}$ in the DC operator. For numerical reasons, it is preferable first to perform the following nonunitary transformation of the large- and small-component bispinors

$$
\left[\begin{array}{c}
\psi^{\mathrm{L}} \\
\psi^{\mathrm{S}}
\end{array}\right]=\left[\begin{array}{cc}
I_{2 \times 2} & 0_{2 \times 2} \\
0_{2 \times 2} & \frac{1}{c_{0}} I_{2 \times 2}
\end{array}\right]\left[\begin{array}{c}
\psi^{\mathrm{L}} \\
\phi^{\mathrm{S}}
\end{array}\right],
$$

which, in the nonrelativistic limit $\left(c_{0} \rightarrow \infty\right)$, yields the following four-component nonrelativistic Hamiltonian for an electron in the external potential $V$ : 
$\hat{h}_{\mathrm{LL}}=\left[\begin{array}{cc}\hat{V} & \boldsymbol{\sigma} \cdot \boldsymbol{\pi} \\ \boldsymbol{\sigma} \cdot \boldsymbol{\pi} & -2 m\end{array}\right]$,

proposed by Lévy-Leblond [53]. At the same time, the twoelectron DC equation factorizes into four equations, one of which is equivalent to the two-electron Schrödinger equation [53-56].

The large- and small-component bispinors in Eq. 16 are typically expanded in separate AO basis sets. However, to obtain the correct nonrelativistic limit, the basis set spanning the small-component bispinors must be related to the large-component basis by the kinetic-balance condition [57]

$\left\{\chi_{\mu}^{\mathrm{S}}\right\} \subseteq\left\{(\boldsymbol{\sigma} \cdot \pi) \chi_{\mu}^{\mathrm{L}}\right\}$,

where $\chi_{\mu}$ is a Cartesian or solid-harmonic Gaussian basis function. If this mapping is one-to-one, the basis set is of the restricted-kinetic-balance (RKB) type. By introducing external vector potentials A representing magnetic fields through the minimal electromagnetic coupling, this mapping is modified since $\pi$ depends linearly on the vector potential. The use of RKB can therefore lead to significant errors for magnetic properties. This problem can be solved by invoking magnetic balance $[58,59]$ or the unrestrictedkinetic-balance (UKB) scheme. In the latter approach, the Cartesian components of the linear momentum $\mathbf{p}$ are treated separately, providing a more flexible basis set to ensure the correct nonrelativistic limit. The difference between the UKB and RKB treatments decreases with increasing quality of the AO basis.

\subsection{Atomic-orbital-basis Kohn-Sham density- functional response theory}

The framework used here to calculate the response functions contributing to Buckingham birefringence has been presented in Refs. [60, 61], which describe a self-consistentfield AO-based response theory for time- and perturbationdependent basis sets. Being formulated in the $\mathrm{AO}$ basis, the approach is transparent to the explicit form of the molecular Hamiltonian and to the parametrization of the self-consistent-field wave function. We have previously utilized this feature to extend the approach to two- and four-component relativistic wave functions for the calculation of higherorder molecular properties involving one-electron operators [35]. Here, this approach is extended further to include KS exchange-correlation (XC) contributions using perturbation-dependent basis sets to first order.

We use as our reference system four-component relativistic and nonrelativistic (Lévy-Leblond) KS wave functions, respectively, obtained by solving the KS equation for the corresponding Hamiltonians, as described in the previous section. To this system, we apply a frequencydependent electromagnetic field represented as

$$
\begin{aligned}
\hat{V}(t)= & {\left[f \exp (\mathrm{i} \omega t)+f^{*} \exp (-\mathrm{i} \omega t)+e\right] \cdot(-\hat{\mu}) } \\
& +\left[q \exp (-\mathrm{i} \omega t)+q^{*} \exp (\mathrm{i} \omega t)+g\right] \cdot(-\hat{\Theta}) \\
& +\left[-\mathrm{i} b \exp (-\mathrm{i} \omega t)+\mathrm{i} b^{*} \exp (\mathrm{i} \omega t)\right] \cdot(-\hat{m}) .
\end{aligned}
$$

In this expression, $f=\left(f_{x}, f_{y}, f_{z}\right)^{\mathrm{T}}$ is the complex electric field, or Jones vector, defining the intensity, polarization and phase of the radiation; $q$ is the complex electric-fieldgradient tensor arising from the radiation; $e, g$ and $-\mathrm{i} b$ are the static electric field, electric field gradient and magnetic field, respectively. The factor $-\mathrm{i}$ has been introduced to ensure real-valued derivative integrals. In our formalism, the complex magnetic field strength $-\mathrm{i} b$ enters the timedependent LAOs defined as [36, 62]

$$
\begin{aligned}
\xi_{\mu}(\mathbf{x})= & \exp \left\{-\frac{\mathrm{i}}{2}\left[\left(-\mathrm{i} b \exp (-\mathrm{i} \omega t)+\mathrm{i} b^{*} \exp (\mathrm{i} \omega t)\right)\right.\right. \\
& \left.\left.\times \mathbf{R}_{\mu}\right] \cdot \mathbf{r}\right\} \chi_{\mu}(\mathbf{x}) .
\end{aligned}
$$

To obtain the working equations for the response functions, we define a quasi-energy gradient of our (non)relativistic wave function with respect to any of the perturbation strengths $w$ in Eq. 20 as [60]

$Q^{w}=\frac{\mathrm{dQ}}{\mathrm{d} w} \stackrel{T_{r_{t}}}{=} \frac{\partial}{\partial w}\left[E(\mathbf{D})-\mathbf{S}^{w} \mathbf{W}\right]$,

where the notation $\stackrel{T r_{t}}{=}$ " indicates that we consider the trace of all matrix expressions and a time average over one period of the applied time-periodic perturbation. In this equation, we have generalized the molecular energy determined by the Hamiltonian in Eq. 13 (or Eq. 18 in the case of the nonrelativistic energy) to read

$$
E(\mathbf{D})=\left(\mathbf{h}+\mathbf{V}(t)-\frac{\mathrm{i}}{2} \mathbf{T}\right) \mathbf{D}+\frac{1}{2} \mathbf{G}^{\gamma}(\mathbf{D}) \mathbf{D}+E_{\mathrm{xc}}(n(\mathbf{D})) .
$$

Here $\mathbf{h}$ and $\mathbf{V}(t)$ are the one-electron AO matrices of the free-particle Dirac operator and the interaction operator in Eq. 20, respectively, whereas the AO matrix elements of the two-electron interaction operator are given as

$\mathbf{G}_{\mu v}^{\gamma}(\mathbf{M})=\sum_{\kappa \lambda} M_{\lambda \kappa}\left(g_{\mu v \kappa \lambda}-\gamma g_{\mu \lambda \kappa v}\right)$,

where $0 \leq \gamma \leq 1$ determines the amount of exact exchange in the calculation (zero in pure KS theory, fractional in hybrid theories, and one in HF theory); for ease of notation, the superscript indicating this scaling is omitted in the following. In Eqs. 22 and 23 we have also introduced 
$T_{\mu v}=\left\langle\dot{\xi}_{\mu} \mid \xi_{v}\right\rangle-\left\langle\xi_{\mu} \mid \dot{\xi}_{v}\right\rangle$,

$S_{\mu \nu}^{w}=\frac{\mathrm{d}}{\mathrm{d} w}\left\langle\xi_{\mu} \mid \xi_{v}\right\rangle$

$\mathbf{W}=\mathbf{D F D}+\frac{\mathrm{i}}{2} \dot{\mathbf{D S D}}-\frac{\mathrm{i}}{2} \mathbf{D S} \dot{\mathbf{D}}$,

and the XC energy $E_{\mathrm{xc}}(n(\mathbf{D}))$ calculated from the density $n(\mathbf{D})$. In Eq. 27, the generalized Fock matrix $\mathbf{F}$ is defined as the partial derivative of the energy functional in Eq. 23 with respect to the transposed density matrix

$\mathbf{F}=\frac{\partial E(\mathbf{D})}{\partial \mathbf{D}^{\mathrm{T}}}=\mathbf{h}+\mathbf{V}(t)-\frac{\mathrm{i}}{2} \mathbf{T}+\mathbf{G}(\mathbf{D})+\mathbf{F}_{\mathrm{xc}}$,

where the $\mathrm{XC}$ contribution to the Fock matrix is given as

$F_{\mathrm{xc}, \mu v}=\int \mathrm{d} \mathbf{r} \Omega_{\mu v}(\mathbf{r}) v_{\mathrm{xc}}(\mathbf{r})=\int \mathrm{d} \mathbf{r} \Omega_{\mu v}(\mathbf{r}) \frac{\delta E_{\mathrm{xc}}}{\delta n(\mathbf{r})}$.

Here, the XC potential $v_{\mathrm{xc}}(\mathbf{r})$ is the functional derivative of the XC energy with respect to the density, $\Omega_{\mu v}(\mathbf{r})$ is the overlap distribution of two AOs, $\xi_{\mu}(\mathbf{r})$ and $\xi_{\nu}(\mathbf{r})$, and integration is over all space. We have assumed the adiabatic approximation, taking the $\mathrm{XC}$ kernel to be time independent $[63,64]$.

To derive the working equations for Buckingham birefringence, we use the quasi-energy Lagrangian in Eq. 22 defined in terms of the magnetic-field perturbation $b$, differentiating this twice with respect to an electric-field perturbation, yielding [22]

$$
\begin{aligned}
Q_{\omega,-\omega, 0}^{b f e} \stackrel{T r_{t}}{=}\left[\mathbf{V}_{\omega,-\omega}^{b f}+\mathbf{F}_{\mathrm{xc} ; \omega,-\omega}^{b f}\right] \mathbf{D}_{0}^{e}+\left[\mathbf{V}_{\omega, 0}^{b e}+\mathbf{F}_{\mathrm{xc} ; \omega, 0}^{b e}\right] \mathbf{D}_{-\omega}^{f} \\
+\left[\mathbf{h}_{\omega}^{b}+\mathbf{V}_{\omega}^{b}-\frac{\mathrm{i}}{2} \mathbf{T}_{\omega}^{b}+\mathbf{F}_{\mathrm{xc} ; \omega}^{b}\right] \mathbf{D}_{-\omega, 0}^{f e} \\
+\left[\mathbf{G}_{\omega}^{b}(\mathbf{D})+E_{\mathrm{xc} ; \omega}^{2, b}(\mathbf{D})\right] \mathbf{D}_{-\omega, 0}^{f e} \\
+\left[\mathbf{G}_{\omega}^{b}\left(\mathbf{D}_{0}^{e}\right)+E_{\mathrm{xc} ; \omega}^{2, b}\left(\mathbf{D}_{0}^{e}\right)\right] \mathbf{D}_{-\omega}^{f}-\mathbf{S}_{\omega}^{b} \mathbf{W}_{-\omega, 0}^{f e}
\end{aligned}
$$

$Q_{\omega,-\omega, 0}^{b_{\beta} f_{\alpha} e_{\gamma}}=J_{\alpha, \beta, \gamma}^{\prime}(\omega)=\mathrm{i}\left\langle\left\langle\hat{\mu}_{\alpha} ; \hat{m}_{\beta}, \hat{\mu}_{\gamma}\right\rangle\right\rangle_{\omega, 0}$.

We have here introduced the notation

$$
\begin{aligned}
\left(E_{x c}^{2, b}\right)_{\mu v}(\mathbf{M}) & =\sum_{\kappa \lambda}\left(E_{x c}^{2, b}\right)_{\mu v \kappa \lambda} M_{\kappa \lambda}, \\
\left(E_{x c ;-\omega}^{2, b}\right)_{\mu v \kappa \lambda} & =\frac{\partial^{2} E_{x c}^{b}}{\partial D_{\mu \nu}^{T} \partial D_{\kappa \lambda}^{T}} \\
& =\int \mathrm{d} \mathbf{r} \Omega_{\mu v}^{b}(\mathbf{r})\left[\int \mathrm{d} \mathbf{r}^{\prime} \Omega_{\kappa \lambda}\left(\mathbf{r}^{\prime}\right) \frac{\partial v_{x c}(\mathbf{r})}{\partial n\left(\mathbf{r}^{\prime}\right)}\right],
\end{aligned}
$$

where we have used the fact that $f$ only enters $\mathbf{V}$, and only linearly. We have also exploited the fact that, when we take the trace of the resulting matrices, the following equality holds:
$\operatorname{Tr}\left[\mathbf{G}\left(\mathbf{D}^{\mathrm{A}}\right) \mathbf{D}^{\mathrm{B}}\right]=\operatorname{Tr}\left[\mathbf{G}\left(\mathbf{D}^{\mathrm{B}}\right) \mathbf{D}^{\mathrm{A}}\right]$.

The quantity $\mathbf{W}_{-\omega, 0}^{f e}$ in Eq. 30 is given by the expression [22]

$$
\begin{aligned}
\mathbf{W}_{-\omega, 0}^{f e}= & \mathbf{D}_{0}^{e} \mathbf{F}_{-\omega}^{f} \mathbf{D}+\mathbf{D}_{0}^{e} \mathbf{F D}_{-\omega}^{f}+\mathbf{D F}_{0}^{e} \mathbf{D}_{-\omega}^{f}-\frac{\omega}{2} \mathbf{D}_{0}^{e} \mathbf{S D}_{-\omega}^{f} \\
& +\mathbf{D F}_{-\omega}^{f} \mathbf{D}_{0}^{e}+\mathbf{D}_{-\omega}^{f} \mathbf{F D}_{0}^{e}+\mathbf{D}_{-\omega}^{f} \mathbf{F}_{0}^{e} \mathbf{D}+\frac{\omega}{2} \mathbf{D}_{-\omega}^{f} \mathbf{S D}_{0}^{e} \\
& +\mathbf{D}_{-\omega, 0}^{f e} \mathbf{F D}+\mathbf{D} \mathbf{F}_{-\omega, 0}^{f e} \mathbf{D}+\mathbf{D} \mathbf{F} \mathbf{D}_{-\omega, 0}^{f e}+\frac{\omega}{2} \mathbf{D}_{-\omega, 0}^{f e} \mathbf{S D} \\
& -\frac{\omega}{2} \mathbf{D} \mathbf{S} \mathbf{D}_{-\omega, 0}^{f e}
\end{aligned}
$$

since the AOs do not depend on the electric-field perturbations $e$ and $f$. In Eq. 35 , both $\mathbf{D}_{-\omega}^{f}$ and $\mathbf{D}_{-\omega, 0}^{f e}$ carry a phase factor $\exp (\mathrm{i} \omega t)$ and hence $\mathrm{i} \dot{\mathbf{D}}^{f}=-\omega \mathbf{D}_{-\omega}^{f}$, whereas $\mathbf{D}_{0}^{e}$ is static and hence $\dot{\mathbf{D}}^{e}=\mathbf{0}$. Although all matrices in Eq. 30 carry a time-dependent exponential phase factor, these cancel, making time averaging redundant. The integrals in $\mathbf{V}_{\omega,-\omega}^{b f}$ are given as $[22,65,66]$

$V_{\mu \nu}^{b_{\beta} f_{\alpha}}=Q_{M N}\left\langle\chi_{\mu}\left|r_{\beta} r_{\alpha}\right| \chi_{v}\right\rangle$,

where $r_{\beta}$ refers to the $\beta$ component of the electron position operator in the phase of the LAO, whereas $r_{\alpha}$ is the $\alpha$ component of the position operator in the electric-dipole moment operator. The $Q_{M N}$ are the elements of an antisymmetric matrix containing the differences between the centers of the AOs $\chi_{\mu}$ and $\chi_{\nu}$ :

$Q_{M N}=\frac{1}{2}\left[\begin{array}{ccc}0 & -Z_{M N} & Y_{M N} \\ Z_{M N} & 0 & -X_{M N} \\ -Y_{M N} & X_{M N} & 0\end{array}\right]$.

If we do not employ LAOs, all contributions that involve derivatives of integrals with respect to the external magnetic field vanish in Eq. 30, yielding a simpler expression for the quadratic response function:

$Q_{\omega,-\omega, 0}^{b f f, \mathrm{NoLAO}} \stackrel{\operatorname{Tr}_{t}}{=}\left(\mathbf{V}_{\omega}^{b}+\mathbf{F}_{x c ; \omega}^{b}\right) \mathbf{D}_{-\omega, 0}^{f e}$.

Since the AOs do not have an explicit dependence on any of the perturbing operators in $\mathscr{B}_{\alpha, \beta \gamma, \delta}$, this tensor is easily obtained from Eq. 38 by replacing $b$ with $q$ :

$Q_{\omega,-\omega, 0}^{q f e} \stackrel{\operatorname{Tr}_{t}}{=}\left(\mathbf{V}_{\omega}^{q}+\mathbf{F}_{x c ; \omega}^{q}\right) \mathbf{D}_{-\omega, 0}^{f e}$,

$Q_{\omega,-\omega, 0}^{q_{\beta, j} f_{\alpha} e_{\delta}}=\mathscr{B}_{\alpha, \beta \gamma, \delta}(\omega)=\left\langle\left\langle\hat{\mu}_{\alpha} ; \hat{\Theta}_{\beta \gamma}, \hat{\mu}_{\delta}\right\rangle\right\rangle_{\omega, 0}$.

In the same manner, $B_{\alpha \beta, \gamma \delta}$ is obtained by replacing $b$ with $g$, and $e$ with $f^{*}$, respectively:

$Q_{0,-\omega, \omega}^{g f f^{*}} \stackrel{T r_{t}}{=}\left(\mathbf{V}_{0}^{g}+\mathbf{F}_{x c ; 0}^{g}\right) \mathbf{D}_{-\omega, \omega}^{f^{*}}$, 
$Q_{0,-\omega, \omega}^{g_{\gamma \gamma} f_{\alpha} f_{\beta}^{*}}=B_{\alpha \beta, \gamma \delta}(\omega)=\left\langle\left\langle\hat{\mu}_{\alpha} ; \hat{\mu}_{\beta}, \hat{\Theta}_{\gamma \delta}\right\rangle\right\rangle_{\omega, 0}$.

For completeness, we note that the polarizability tensor given in Eq. 4 can be evaluated as

$Q_{-\omega ; \omega}^{f^{*} f} \stackrel{T r_{t}}{=} \mathbf{V}_{-\omega}^{f^{*}} \mathbf{D}_{\omega}^{f}$.

The expressions given here are almost the same as those recently presented in Ref. [22], with the exception of the additional XC contributions. However, the current equations have been derived on the basis of a four-component relativistic DC Hamiltonian. It is the generic nature of the AO-based response theory that allows us to calculate molecular properties in a manner that is transparent to the underlying reference wave function.

The evaluation of the $\mathrm{XC}$ functional derivatives has previously been described in the context of perturbationindependent basis sets by Sałek et al. [67] and, in the specific case of magnetic-field perturbed densities, by Krykunov et al. [68] and by Kjærgaard et al. [69]. A general strategy for the evaluation of higher-order perturbed XC energies and functionals is given in Ref. [60]. Extensions to include spin-density contributions have furthermore been described [70], also in conjunction with the use of perturbation-dependent basis sets [61].

To develop an implementation suitable for higher-order mixed-field XC response contributions, we have found it convenient to evaluate the $\mathrm{XC}$ terms in Eq. 30 in two steps. First, we calculate the magnetic-field derivatives $n^{e b}, \nabla$ $n^{e b}$, and $\left(\nabla n \cdot \nabla n^{e}\right)^{b}$ of the perturbed density variables $n^{e}, \nabla n^{e}$, and $\left(\nabla n \cdot \nabla n^{e}\right)$, where $e$ represents an electricfield perturbation. Next, this set of magnetic-field derivatives is used as input variables to conventional (possibly LAO-unaware) response modules that form the required matrix elements without the need for additional programming. The functional derivatives needed for constructing the XC matrix elements are obtained using automatic differentiation [71].

We finally outline the procedure for determining the first- and second-order perturbed density matrices $\mathbf{D}^{b}$ and $\mathbf{D}^{f e}$ that appear in the equations above. The perturbed density matrix of order $n$ with respect to a set of perturbations $w$ can be separated into particular and homogeneous components

$\mathbf{D}^{w_{n}}=\mathbf{D}_{P}^{w_{n}}+\mathbf{D}_{H}^{w_{n}}$.

The particular component $\mathbf{D}_{P}^{w_{n}}$ can be calculated from a knowledge of the lower-order (perturbed) density matrices through the equation [60]

$\mathbf{D}_{P}^{w_{n}} \mathbf{S}^{\dagger} \mathbf{D}^{\dagger}-(\mathbf{1}-\mathbf{D S}) \mathbf{D}_{P}^{w_{n}}=-(\mathbf{D S D})_{n-1}^{w_{n}}$,

where the subscript $n-1$ on the right-hand side indicates that only perturbed density matrices of order $n-1$ are included in the total derivative. The particular component can thus be calculated from known quantities.

The homogeneous component of the perturbed density matrix $\mathbf{D}_{H}^{w_{n}}$ in Eq. 44 is determined iteratively from the equation

$\mathbf{D}_{H}^{w_{n}} \mathbf{S}^{\dagger} \mathbf{D}^{\dagger}-(\mathbf{1}-\mathbf{D S}) \mathbf{D}_{H}^{w_{n}}=\mathbf{0}$.

As in our previous study of nonlinear electric responses in the relativistic domain [35], we determine the homogeneous component of the perturbed densities by solving a linear set of equations of the general form [60]

$\left(\mathbf{E}^{[2]}-\omega \mathbf{S}^{[2]}\right) \mathbf{X}^{w_{n}}=\mathbf{M}_{n-1}^{\left[w_{1} w_{2} \cdots\right]}$.

In this equation, we have introduced the generalized electronic Hessian $\mathbf{E}^{[2]}$ and metric $\mathbf{S}^{[2]}$ matrices, as well as a response matrix $\mathbf{X}^{w_{n}}$, which determines the unique elements of the homogeneous component of the perturbed density matrix through

$\mathbf{D}_{H}^{w_{n}}=\mathbf{D S X}^{w_{n}}-\mathbf{X}^{w_{n}} \mathbf{S}^{\dagger} \mathbf{D}^{\dagger}$.

In Eq. $47, \mathbf{M}_{n-1}^{\left[w_{1} w_{2} \cdots\right]}$ is a general right-hand-side vector that involves a collection of perturbation and (lower-order) perturbed density matrices; for details, see Ref. [60].

Whereas our expression for the quasi-energy derivatives has been formulated in the AO basis, we do not solve the linear sets of equations in this basis. Instead, we transform the right-hand side of Eq. 47 to the four-component spinor basis and use the linear response solver of Saue and Jensen [72]. In this manner, we ensure that the electronic Hessian remains diagonally dominant, thereby improving convergence. The transformation to the spinor basis also allows us to utilize quaternion algebra in the solution of the linear equations in Eq. 47, as described by Saue and Jensen [72, 73]. The response vectors obtained are subsequently transformed back to the $\mathrm{AO}$ basis and converted into the homogeneous components of the perturbed density matrix, which is used in the calculation of the response functions that determine the Buckingham birefringence. For details, we refer to the literature describing the various aspects of the procedure $[35,60,72]$.

\section{Computational details}

All Buckingham-birefringence results have been obtained using a development version of the DIRAC program package [74]. The relativistic calculations have been carried out employing the four-component DC Hamiltonian; for the nonrelativistic reference values, we have used the Lévy-Leblond Hamiltonian [53].

In addition to the HF method, we have employed the KS method with the LDA (SVWN5) [75, 76], BLYP [77-79], 
B3LYP [80, 81], PBE [82], and PBE0 [83] XC functionals. These are nonrelativistic functionals which, with the DC Hamiltonian, have been evaluated using relativistic densities and density gradients. We have employed the full derivatives of the functionals provided by the XCFun library [71, 84]. Spin-density contributions [70] to XC matrix elements have been ignored.

Experimental equilibrium geometries from the compilation in Ref. [85] were used for $\mathrm{CO}_{2}\left(R_{\mathrm{CO}}=2.19169 a_{0}\right)$ and $\mathrm{CS}_{2}\left(R_{\mathrm{CS}}=2.93391 a_{0}\right)$. For $\mathrm{CSe}_{2}$, we have used the experimental bond length of $R_{\mathrm{CSe}}=3.19993 a_{0}$ reported in Ref. [86]. For $\mathrm{CTe}_{2}$, we have calculated the bond length of $R_{\mathrm{CTe}}=3.60056 a_{0}$ using the Gaussian 09 package [87] with the Ahlrichs def2-TZVPP basis [88] for C and Te in combination with the Stuttgart/Dresden 28-electron effective core pseudopotential [89] and the B3LYP XC functional.

We have used the u-aug-cc-pVDZ and u-aug-cc-pVTZ ("u-" meaning uncontracted) basis sets of Dunning [90, 91] for $\mathrm{C}, \mathrm{O}$ and $\mathrm{S}$, and the augmented all-electron $\mathrm{u}-\mathrm{DZ}$ and $\mathrm{u}-\mathrm{TZ}$ basis sets of Dyall $[92,93]$ for Se and Te. The small-component basis for the DC calculations has been generated using UKB, with RKB imposed in the canonical orthonormalization step [45]. In the self-consistent-field and response calculations, the small-component two-electron Coulomb integrals (SSISS) have been approximated using a point-charge model [94]. A Gaussian charge distribution has been chosen as the nuclear model in the relativistic and nonrelativistic calculations, using the recommended values in Ref. [95].

\section{Results}

\subsection{The temperature-independent part} of the Buckingham birefringence

We have collected our results for $b(\omega)$, the temperatureindependent contribution to Buckingham birefringence, for the series $\mathrm{CX}_{2}(\mathrm{X}=\mathrm{O}, \mathrm{S}, \mathrm{Se}, \mathrm{Te})$ using the DC Hamiltonian for a variety of different $\mathrm{XC}$ functionals and basis sets in Table 1. To assess the effect of field dependence in the basis functions, we report results using conventional as well as London AOs. In this table, the coupled-cluster results for $\mathrm{CO}_{2}$ and $\mathrm{CS}_{2}$ in the large field-independent d-aug-cc-pVQZ basis [17] are listed, together with the available experimentally derived data, taken from Refs. [29, 30] for $\mathrm{CO}_{2}$ and from Ref. [30] for $\mathrm{CS}_{2}$. In Ref. [34], the authors performed a single-temperature $(T=298 \mathrm{~K})$ measurement of the Buckingham birefringence of $\mathrm{CSe}_{2}$, obtaining an estimate for the infinite-dilution Buckingham constant ${ }_{\mathrm{m}} Q$ by assuming $b(\omega)$ to be negligible, vide infra.

The first thing to note from Table 1 is the importance of introducing field dependence in the AOs-in particular, in the smallest $\mathrm{u}$-aug-DZ basis. For $\mathrm{CO}_{2}$ and $\mathrm{CS}_{2}$, the $\mathrm{LAO}$ field dependence induces changes as large as $25-30 \%$, largely independent of the choice of XC functional. Going down in the $\mathrm{CX}_{2}$ series, the effect of field dependence decreases, being only $10-15 \%$ for $\mathrm{CTe}_{2}$ in the u-aug-DZ basis. In the larger u-aug-TZ basis, the effect of LAOs is smaller, being on average $5-10 \%$ for the entire series, the largest effect being once again observed for the lightest members of the series. The changes observed in the LAO results when the basis is increased from u-aug-DZ to u-aug-TZ are small, about $5 \%$ for $\mathrm{CO}_{2}$ and less than $1-2 \%$ for $\mathrm{CS}_{2}, \mathrm{CSe}_{2}$ and $\mathrm{CTe}_{2}$. The observed importance of using LAOs for rapid basis-set convergence corroborates the findings of our recent nonrelativistic study [22]. Please note that the $b(\omega)$ term for the studied series $\mathrm{CX}_{2}(\mathrm{X}=\mathrm{O}, \mathrm{S}$, $\mathrm{Se}, \mathrm{Te})$ is gauge-origin independent by symmetry.

Given that the relativistic correction to the temperatureindependent part of Buckingham birefringence is negligible for $\mathrm{CO}_{2}$ (vide infra), we can compare our $\mathrm{CO}_{2}$ results directly with the high-level coupled-cluster singles-anddoubles (CCSD) results of Ref. [17]. As this study employed the large d-aug-cc-pVQZ basis, these CCSD results are expected to be reasonably close to the basis-set limit. Prior to this work, there have been three Buckingham-birefringence studies using KS theory, but only with field-independent basis sets [18-20]. Having established the importance of LAOs in Ref. [22] and in Table 1, the qualities of different $\mathrm{XC}$ functionals can now be more reliably assessed.

As seen from Table 1, the effect of electron correlation on $b(\omega)$ is significant, with changes from HF theory to CCSD theory of about $13 \%$ for $\mathrm{CO}_{2}$ and $15 \%$ for $\mathrm{CS}_{2}$. Interestingly, whereas electron correlation increases the magnitude of the temperature-independent contribution to the Buckingham birefringence of $\mathrm{CO}_{2}$, the opposite happens for $\mathrm{CS}_{2}$. Moreover, the $\mathrm{CO}_{2}$ and $\mathrm{CS}_{2}$ results obtained with different $\mathrm{XC}$ functionals do not lead to any clear conclusions regarding their ability to capture the effect of electron correlation on $b(\omega)$. For $\mathrm{CO}_{2}$, all functionals overestimate the effect of correlation; the hybrid functionals B3LYP and PBE0 perform best, the PBE0 value being very close to the CCSD value. For $\mathrm{CS}_{2}$, all $\mathrm{XC}$ functionals perform poorly, typically recovering less than one third of the total correlation effect as calculated using CCSD theory, the PBE0 functional again providing the best $\mathrm{KS}$ result.

Because of the very large experimental error bars, comparison with experimental results does not provide a stringent test on the different computational methods-all calculated values fall comfortably within three standard deviations from the center of the experimental distribution for $\mathrm{CO}_{2}$ and $\mathrm{CS}_{2}$. As discussed for linear birefringences elsewhere [8-11], this difficulty arises from the extreme 
Table 1 The value of $b(\omega)$ calculated using the DC Hamiltonian (atomic units)

\begin{tabular}{|c|c|c|c|c|c|c|c|}
\hline & & & $\begin{array}{l}\text { u-aug-DZ } \\
\text { CGO }\end{array}$ & $\begin{array}{l}\text { u-aug-DZ } \\
\text { LAO }\end{array}$ & $\begin{array}{l}\text { u-aug-TZ } \\
\text { CGO }\end{array}$ & $\begin{array}{l}\text { u-aug-TZ } \\
\text { LAO }\end{array}$ & $\begin{array}{l}\text { d-aug-QZ } \\
\text { CGO }\end{array}$ \\
\hline $\mathrm{CO}_{2}$ & $\mathrm{HF}$ & & -34.7 & -46.7 & -43.6 & -48.3 & \\
\hline \multirow[t]{8}{*}{$\lambda=632.8 \mathrm{~nm}$} & LDA & & -40.5 & -56.3 & -51.4 & -58.9 & \\
\hline & BLYP & & -41.7 & -59.1 & -52.4 & -61.5 & \\
\hline & B3LYP & & -39.2 & -54.9 & -49.5 & -57.1 & \\
\hline & PBE & & -41.3 & -57.7 & -51.5 & -59.9 & \\
\hline & PBE0 & & -38.6 & -53.0 & -48.3 & -55.0 & \\
\hline & $\mathrm{CCSD}^{\mathrm{a}}$ & & & & & & -54.48 \\
\hline & $\exp ^{b}$ & $-160 \pm 80$ & & & & & \\
\hline & $\exp ^{c}$ & $-100 \pm 200$ & & & & & \\
\hline $\mathrm{CS}_{2}$ & $\mathrm{HF}$ & & -380.4 & -467.6 & -462.8 & -482.5 & \\
\hline \multirow[t]{7}{*}{$\lambda=632.8 \mathrm{~nm}$} & LDA & & -353.3 & -455.5 & -435.8 & -464.1 & \\
\hline & BLYP & & -352.5 & -474.2 & -433.8 & -476.1 & \\
\hline & B3LYP & & -355.6 & -462.0 & -436.3 & -468.3 & \\
\hline & PBE & & -356.3 & -460.6 & -428.7 & -462.4 & \\
\hline & PBE0 & & -361.7 & -450.7 & -432.3 & -456.2 & \\
\hline & $\operatorname{CCSD}^{\mathrm{a}}$ & & & & & & -410.91 \\
\hline & $\exp ^{b}$ & $-1,200 \pm 800$ & & & & & \\
\hline $\mathrm{CSe}_{2}$ & $\mathrm{HF}$ & & -661.8 & -766.4 & -754.4 & -781.5 & \\
\hline \multirow[t]{6}{*}{$\lambda=632.8 \mathrm{~nm}$} & LDA & & -603.8 & -722.3 & -695.4 & -728.9 & \\
\hline & BLYP & & -590.6 & -737.6 & -682.1 & -731.4 & \\
\hline & B3LYP & & -606.9 & -733.3 & -699.1 & -737.5 & \\
\hline & PBE & & -614.7 & -738.7 & -692.2 & -733.5 & \\
\hline & PBE0 & & -633.6 & -737.1 & -707.3 & -738.1 & \\
\hline & $\exp ^{d}$ & $\approx 0$ & & & & & \\
\hline $\mathrm{CTe}_{2}$ & $\mathrm{HF}$ & & $-1,294.1$ & $-1,404.1$ & $-1,798.4$ & $-1,839.4$ & \\
\hline \multirow[t]{5}{*}{$\lambda=632.8 \mathrm{~nm}$} & LDA & & -981.6 & $-1,127.9$ & $-1,283.4$ & $-1,331.9$ & \\
\hline & BLYP & & -757.1 & -938.6 & $-1,126.0$ & $-1,196.9$ & \\
\hline & B3LYP & & $-1,441.3$ & $-1,599.3$ & $-1,690.8$ & $-1,745.8$ & \\
\hline & PBE & & -982.3 & $-1,137.5$ & $-1,299.1$ & $-1,358.9$ & \\
\hline & PBE0 & & $-1,495.3$ & $-1,626.6$ & $-1,697.9$ & $-1,743.3$ & \\
\hline $\mathrm{CTe}_{2}$ & $\mathrm{HF}$ & & $-1,379.6$ & $-1,507.0$ & $-1,419.8$ & $-1,461.9$ & \\
\hline \multirow[t]{5}{*}{$\lambda=694.3 \mathrm{~nm}$} & LDA & & $-1,076.5$ & $-1,223.7$ & $-1,395.0$ & $-1,442.3$ & \\
\hline & BLYP & & -917.6 & $-1,101.4$ & $-1,330.0$ & $-1,399.4$ & \\
\hline & B3LYP & & -939.1 & $-1,098.9$ & $-1,268.7$ & $-1,324.1$ & \\
\hline & PBE & & $-1,070.6$ & $-1,227.7$ & $-1,401.8$ & $-1,460.4$ & \\
\hline & PBE0 & & $-1,075.1$ & $-1,207.9$ & $-1,307.2$ & $-1,353.0$ & \\
\hline
\end{tabular}

For comparison, the CCSD values from Ref. [17] and the experimental values from Refs. [29, 30, 34] have been included. For C, O and S we have used the u-aug-cc-pVDZ and u-aug-cc-pVTZ ("u-" meaning uncontracted) basis sets of Dunning [90, 91], for Se and Te the augmented allelectron u-DZ and u-TZ basis sets of Dyall [92, 93]. CGO means that a common gauge origin (placed at the carbon nucleus) has been employed

${ }^{a}$ Ref. [17]

${ }^{b}$ Ref. [30]

${ }^{c}$ Ref. [29]

${ }^{\mathrm{d}}$ Ref. [34], the value assumed in the derivation of the quadrupole moment from a single-temperature infinite-dilution measurement.

sensitivity of the infinite-temperature extrapolation performed on the experimental data to estimate $b(\omega)$. From comparison with experimentally derived data, it is therefore not possible to draw definite conclusions regarding the reliability of the various $\mathrm{XC}$ functionals for the mixed hyperpolarizabilities that determine the 
temperature-independent contribution to Buckingham birefringence. Note, however, that the $b(\omega)$ contribution for $\mathrm{CSe}_{2}$ is computed to be of the order of -700 a.u. We shall later return to the consequences that this computed value has for the estimate of the quadrupole moment of $\mathrm{CSe}_{2}$ made by Brereton and co-workers in Ref. [34].

In Table 2, we have collected the individual temperature-independent contributions to the Buckingham birefringence (see Eq. 3) calculated in the u-aug-TZ basis with LAOs, using both the relativistic DC Hamiltonian and the nonrelativistic Lévy-Leblond Hamiltonian. The dominant (negative) temperature-independent contribution to Buckingham birefringence is the electric-dipole-electric-dipolemagnetic-dipole hyperpolarizability $J_{\alpha, \beta, \gamma}^{\prime}$ term, which according to Eq. 5 enters $b(\omega)$ multiplied by $-2 / 3 \omega$. The $B_{\alpha \beta, \alpha \beta}$ and $\mathscr{B}_{\alpha, \alpha \beta, \beta}$ terms are much larger but nearly cancel, entering $b(\omega)$ as $(2 / 15)\left(B_{\alpha \beta, \alpha \beta}-\mathscr{B}_{\alpha, \alpha \beta, \beta}\right)$ and typically contributing about $10 \%$ to $b(\omega)$.

As expected, the relativistic correction to $b(\omega)$ is dominated by the correction to $J_{\alpha, \beta, \gamma}^{\prime}$. The correction is fairly small, however, even for $\mathrm{CSe}_{2}$, thus leaving the resulting temperature-independent Buckingham birefringence virtually unaffected by relativity for the three lightest members of the $\mathrm{CX}_{2}$ series. Further studies are needed to establish whether this is a general feature of $b(\omega)$, valid also for polar systems, for example, or whether this insensitivity to relativity is unique to the $\mathrm{CX}_{2}$ series. The relativistic corrections to $B_{\alpha \beta, \alpha \beta}$ and $\mathscr{B}_{\alpha, \alpha \beta, \beta}$ are small in relative terms, being only about $2 \%$ for $\mathrm{CSe}_{2}$. By cancellation, the total relativistic correction from $B_{\alpha \beta, \alpha \beta}-\mathscr{B}_{\alpha, \alpha \beta, \beta}$ is even smaller, being less than one percent for all $\mathrm{XC}$ functionals.

The relativistic effects vary significantly with the choice of XC functional-in particular, for the heavier elements. It is noteworthy that the use of exact exchange (in HF and hybrid theories) gives a negative relativistic correction for $\mathrm{CTe}_{2}$ at $\lambda=632.8 \mathrm{~nm}$, whereas pure $\mathrm{KS}$ theory gives a positive and much larger relativistic correction. For $\mathrm{CTe}_{2}$, we note from Table 2 that the relativistic correction becomes substantial for $b(\omega)$ at $\lambda=632.8 \mathrm{~nm}$, amounting to $30 \%$ for the BLYP functional.

The reason for the much larger relativistic corrections in $\mathrm{CTe}_{2}$ is the presence of a low-lying ${ }^{3} \Sigma_{u}^{+}$state (scalar relativistic notation), rather close in energy to the frequency of the applied field. Whereas the transition to this state is dipole forbidden in the nonrelativistic case, it is allowed in the four-component relativistic case due to spin-orbit coupling. The four-component relativistic calculations are thus much more dependent on the predicted excitation energy for this state than are the nonrelativistic calculations, as the approaching electronic resonance may affect the different $\mathrm{XC}$ functionals differently depending on how close the energy of the relevant ${ }^{3} \Sigma_{u}^{+}$state is to the applied laser frequency.

At $\lambda=694.3 \mathrm{~nm}$, the relativistic correction in $\mathrm{CTe}_{2}$ is positive for all employed XC functionals, again with the hybrid functionals standing out and yielding very similar relativistic corrections.

\subsection{The temperature-dependent contribution} to the Buckingham birefringence

In Tables 3 and 4, we have collected the values for the tensors that contribute to the temperature-dependent part of Buckingham birefringence in $\mathrm{CO}_{2}$ to $\mathrm{CSe}_{2}$ and $\mathrm{CTe}_{2}$, respectively, calculated in the u-aug-TZ basis. The contributing tensors are the polarizability (both the isotropic and anisotropic components) and the quadrupole moment along the molecular axis (which is the only unique component of the quadrupole-moment tensor for the linear molecules studied here). The relativistic corrections follow the trend observed for the temperature-independent part but their magnitude is larger, amounting to $5-7 \%$ of the nonrelativistic value for the polarizability anisotropy and quadrupole moment of $\mathrm{CSe}_{2}$.

Interestingly, the relativistic correction to the polarizability of $\mathrm{CTe}_{2}$ is largest in HF theory (Table 4) and positive for both frequencies, whereas the relativistic corrections to the polarizability are negligible for all studied $\mathrm{XC}$ functionals and negative except for the hybrid PBE0 and B3LYP functionals at $\lambda=632.8 \mathrm{~nm}$.

By contrast, the relativistic corrections to the quadrupole moment are substantial, indicating that relativity leads to a significant restructuring of the electron density, increasing the quadrupole moment by $10-15 \%$. Indeed, spin-free calculations confirm that the relativistic increase in the quadrupole moment is almost entirely a scalar relativistic effect. To understand this effect we have compared nonrelativistic and scalar relativistic orbital contributions to the quadrupole moment (data not shown). This analysis shows that the change in the quadrupole moment when including scalar relativity is due to a relativistic contraction of the valence $\sigma$ orbitals related to a contraction of the participating $s$ - and $p$-orbitals. This decreases the electronic contribution to the quadrupole moment and increases the total (electronic + nuclear) quadrupole moment. Clearly, despite being a property that largely probes the outer part of the electron density, the quadrupole moment is strongly dependent on a proper relativistic treatment.

Whereas the isotropic polarizability is fairly insensitive to electron correlation, with the notable exception of $\mathrm{CTe}_{2}$, electron correlation is moderately important for the polarizability anisotropy and quadrupole moment of the lighter members of the series $\left(\mathrm{CO}_{2}, \mathrm{CS}_{2}\right.$ and $\left.\mathrm{CSe}_{2}\right)$, contributing 
Table 2 The temperature-independent contribution to Buckingham-birefringence, $b(\omega)$, and its individual components (isotropic averages, Einstein implicit summation implied) calculated using the u-aug-cc-pVTZ LAO basis and the DC Hamiltonian

\begin{tabular}{|c|c|c|c|c|c|c|}
\hline & & $b(\omega)$ & $J_{\alpha, \beta, \gamma}^{\prime}$ & $B_{\alpha \beta, \alpha \beta}$ & $\mathscr{B}_{\alpha, \alpha \beta, \beta}$ & \\
\hline $\mathrm{CO}_{2}$ & $\mathrm{HF}$ & $-48.3(-0.1)$ & $5.1(0.0)$ & $-1,069.7 \quad(-1.5)$ & $-1,061.2$ & $(-1.5)$ \\
\hline \multirow[t]{5}{*}{$\lambda=632.8 \mathrm{~nm}$} & LDA & $-58.9(-0.1)$ & $6.2(0.0)$ & $-1,641.8 \quad(-2.6)$ & $-1,628.1$ & $(-2.7)$ \\
\hline & BLYP & $-61.5(-0.1)$ & $6.5(0.0)$ & $-1,738.9 \quad(-3.0)$ & $-1,725.9$ & $(-3.0)$ \\
\hline & B3LYP & $-57.1(-0.1)$ & $6.0(0.0)$ & $-1,519.2 \quad(-2.5)$ & $-1,507.1$ & $(-2.5)$ \\
\hline & PBE & $-59.9(-0.1)$ & $6.3(0.0)$ & $-1,676.6 \quad(-2.9)$ & $-1,663.7$ & $(-2.9)$ \\
\hline & PBE0 & $-55.0(-0.1)$ & $5.8(0.0)$ & $-1,432.0 \quad(-2.3)$ & $-1,420.4$ & $(-2.3)$ \\
\hline $\mathrm{CS}_{2}$ & $\mathrm{HF}$ & $-482.5(-1.9)$ & $46.8(0.2)$ & $-11,867.3 \quad(-61.8)$ & $-11,497.1$ & $(-60.8)$ \\
\hline \multirow[t]{5}{*}{$\lambda=632.8 \mathrm{~nm}$} & LDA & $-464.1 \quad(-2.2)$ & $44.1(0.2)$ & $-14,335.1 \quad(-92.3)$ & $-13,914.8$ & $(-91.8)$ \\
\hline & BLYP & $-476.1 \quad(-2.2)$ & $44.9(0.2)$ & $-15,307.2 \quad(-106.7)$ & $-14,857.0$ & $(-106.3)$ \\
\hline & B3LYP & $-468.3(-2.1)$ & $44.6(0.2)$ & $-13,972.1 \quad(-89.6)$ & $-13,554.6$ & $(-88.9)$ \\
\hline & PBE & $-462.4(-2.1)$ & $43.9(0.2)$ & $-14,321.7 \quad(-93.0)$ & $-13,904.1$ & $(-92.5)$ \\
\hline & PBE0 & $-456.2(-2.0)$ & $43.7(0.2)$ & $-12,984.2 \quad(-76.9)$ & $-12,598.8$ & $(-76.1)$ \\
\hline $\mathrm{CSe}_{2}$ & $\mathrm{HF}$ & $-781.5(5.3)$ & $71.9(-1.0)$ & $-22,358.3 \quad(-407.3)$ & $-21,493.6$ & $(-376.4)$ \\
\hline \multirow{5}{*}{$\lambda=632.8 \mathrm{~nm}$} & LDA & $-728.9(-0.7)$ & $66.5(0.2)$ & $-26,054.2 \quad(-571.8)$ & $-25,205.4$ & $(-580.6)$ \\
\hline & BLYP & $-731.4(1.9)$ & $66.0(0.0)$ & $-28,534.5 \quad(-698.4)$ & $-27,635.1$ & $(-713.2)$ \\
\hline & B3LYP & $-737.5(0.8)$ & $67.1(-0.0)$ & $-25,948.1 \quad(-570.3)$ & $-25,078.0$ & $(-574.4)$ \\
\hline & PBE & $-733.5(-0.1)$ & $66.9(0.2)$ & $-26,622.6 \quad(-633.0)$ & $-25,768.0$ & $(-643.4)$ \\
\hline & PBE0 & $-738.1 \quad(-0.8)$ & $67.8(0.1)$ & $-24,002.8 \quad(-500.4)$ & $-23,178.6$ & $(-500.7)$ \\
\hline $\mathrm{CTe}_{2}$ & $\mathrm{HF}$ & $-1,839.4 \quad(-47.1)$ & $-65.4(-210.3)$ & $-78,749.0 \quad(-26,170.6)$ & $-60,414.8$ & $(-11,210.8)$ \\
\hline \multirow[t]{5}{*}{$\lambda=632.8 \mathrm{~nm}$} & LDA & $-1,331.9(245.5)$ & $91.1(-34.5)$ & $-59,784.2 \quad(-4,149.6)$ & $-56,123.0$ & $(-3,593.6)$ \\
\hline & BLYP & $-1,196.9(359.1)$ & $71.9(-47.9)$ & $-66,794.5 \quad(-5,223.1)$ & $-62,808.5$ & $(-4,590.8)$ \\
\hline & B3LYP & $-1,745.8 \quad(-141.2)$ & $129.3(2.9)$ & $-61,634.2 \quad(-4,832.6)$ & $-57,516.6$ & $(-3,973.1)$ \\
\hline & PBE & $-1,358.9(237.1)$ & $93.1(-33.4)$ & $-61,992.8 \quad(-4,592.5)$ & $-58,267.9$ & $(-4,049.3)$ \\
\hline & PBE0 & $-1,743.3 \quad(-100.7)$ & $131.5(-1.6)$ & $-57,060.6 \quad(-4,374.7)$ & $-53,114.7$ & $(-3,510.6)$ \\
\hline $\mathrm{CTe}_{2}$ & $\mathrm{HF}$ & $-1,461.9(300.6)$ & $42.6(-95.8)$ & $-62,159.3 \quad(-11,635.2)$ & $-54,436.8$ & $(-6,591.7)$ \\
\hline \multirow[t]{5}{*}{$\lambda=694.3 \mathrm{~nm}$} & LDA & $-1,442.3(120.1)$ & $105.9(-15.5)$ & $-56,883.4 \quad(-3,393.2)$ & $-54,136.4$ & $(-3,112.1)$ \\
\hline & BLYP & $-1,399.4(160.4)$ & $98.9(-19.8)$ & $-63,238.4 \quad(-4,218.2)$ & $-6,0274.5$ & $(-3,916.0)$ \\
\hline & B3LYP & $-1,324.1(268.9)$ & $89.1(-33.7)$ & $-58,556.7 \quad(-4,017.4)$ & $-55,417.2$ & $(-3,463.5)$ \\
\hline & PBE & $-1,460.4(126.1)$ & $107.1(-15.9)$ & $-58,927.7 \quad(-3,798.3)$ & $-56,132.7$ & $(-3,528.9)$ \\
\hline & PBE0 & $-1,353.0(266.2)$ & $94.0(-33.3)$ & $-54,299.0 \quad(-3,618.3)$ & $-51,312.3$ & $(-3,078.5)$ \\
\hline
\end{tabular}

In parentheses, we report the relativistic correction (the difference between the DC Hamiltonian and a nonrelativistic Hamiltonian). For $\mathrm{C}$, $\mathrm{O}$ and $\mathrm{S}$ we have used the u-aug-cc-pVTZ ("u-" meaning uncontracted) basis sets of Dunning [90, 91], for Se and Te the augmented all-electron u-TZ basis sets of Dyall $[92,93]$. The basis sets employ LAO. All results are given in atomic units

3-5\%. Also in this case, $\mathrm{CTe}_{2}$ displays much larger dependence on correlation at $\lambda=632.8 \mathrm{~nm}$ (by almost $15 \%$ with the BLYP functional), possibly because of the low-lying ${ }^{3} \Sigma_{u}^{+}$ state. Interestingly, the relativistic corrections show only a weak dependence on electron correlation. We also note that the polarizability anisotropy exhibits large correlation effects at $\lambda=632.8 \mathrm{~nm}$, more than $25 \%$ at the relativistic fourcomponent level of theory for the LDA functional.

In Table 3, we report the available experimental reference data for most of the observables involved in Buckingham birefringence of the series of studied molecules. Whereas the electric-dipole polarizability anisotropy of $\mathrm{CO}_{2}$ is reasonably well reproduced (albeit all XC functionals yield values below the center of the experimental distribution, with the PBE, LDA and BLYP functionals performing better than the hybrid PBE0 and B3LYP functionals), the disagreement with experiment is notable for $\mathrm{CS}_{2}$ (where we underestimate $\Delta \alpha$ ) and $\mathrm{CSe}_{2}$ (where we overestimate $\Delta \alpha$ ). For $\mathrm{CO}_{2}$ and $\mathrm{CS}_{2}$, our DFT results lie on the opposite side of experiment relative to the highly accurate $a b$ initio values of Coriani and co-workers in Ref. [17]. Note also the neglect of vibrational corrections, whose magnitude for the heavier members of the series may heavily affect the comparison.

With regard to the quadrupole moment, comparisons can again be made with experiment, with the $\operatorname{CCSD}(\mathrm{T})$ results 


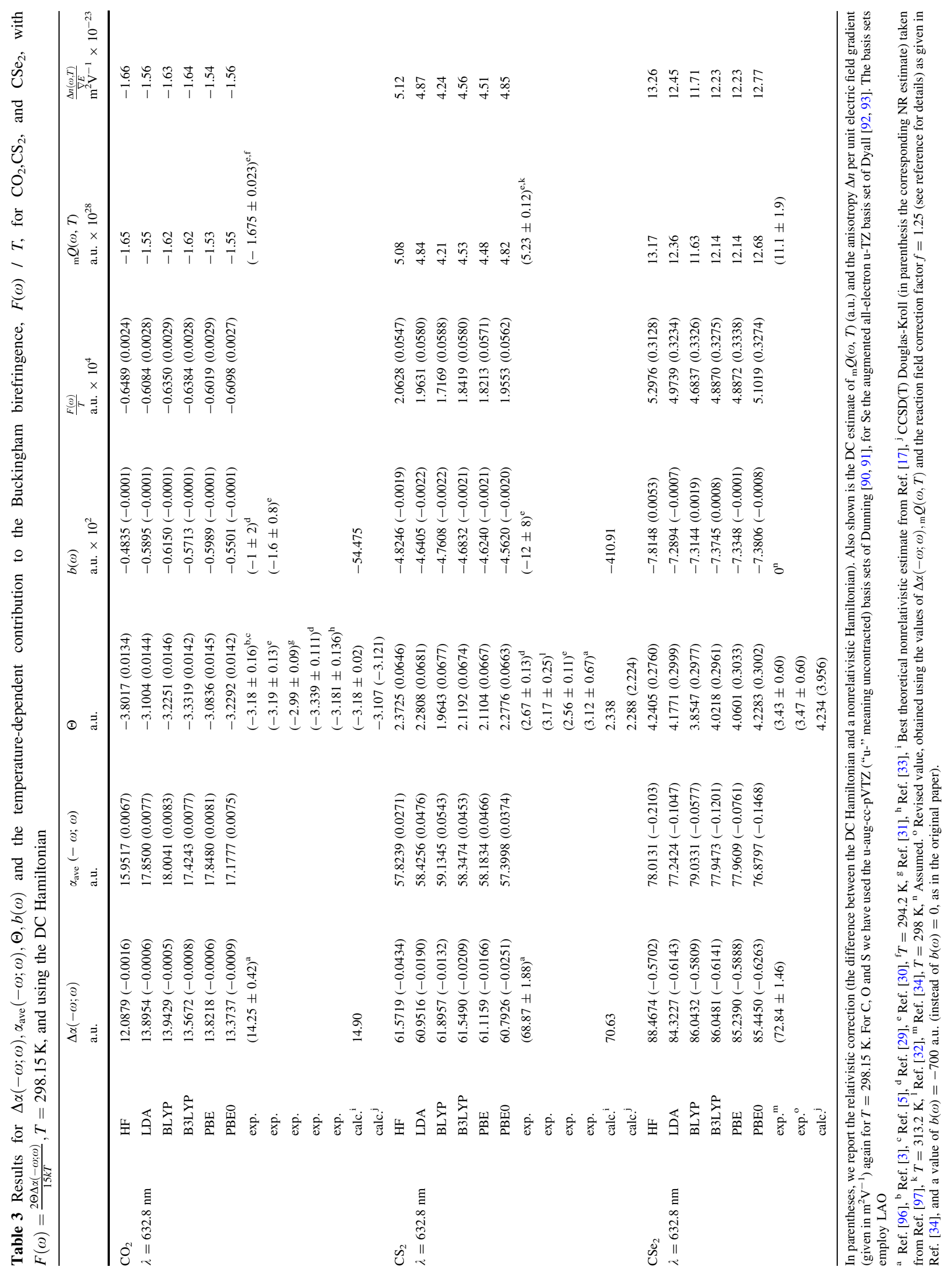


obtained by Benkova and Sadlej [97] employing the HyPolX and HyPolX_dk basis sets and including scalar relativistic effects within the two-component DouglasKroll approximation, and, for $\mathrm{CO}_{2}$ and $\mathrm{CS}_{2}$, with the results of Ref. [17]. Among the XC functionals, the BLYP and PBE0 functionals get closest to the nicely agreeing experimental and $a b$ initio values (both aiming at a value of 3.18 a.u.) for the quadrupole moment of $\mathrm{CO}_{2}$. Again, the reader should consult Ref. [17] for further details-for instance, on the effect of molecular vibrations.

There are several experimental values for the quadrupole moment of $\mathrm{CS}_{2}$. Both $a b$ initio and this relativistic DFT study are closer to the values in the range of 2.6-2.7 a.u. in Refs. [29, 30]. The experimental value for $\mathrm{CSe}_{2}$ was obtained by Brereton and co-workers [34] by infinite-dilution extrapolation of data at $298 \mathrm{~K}$ for Buckingham birefringence observed in carbon tetrachloride, with a laser source at $\lambda=632.8 \mathrm{~nm}$. In deriving the value of $\Theta=3.43 \pm 0.60$ a.u., the authors assumed a negligible $b(\omega)$ term, which we compute instead at a value of about -700 a.u. When, as done in other cases [8-11], this value is inserted in the expression for $\mathrm{m} Q(\omega, T)$ and the quadrupole moment is re-evaluated, assuming for the electric-dipole polarizability anisotropy and the reaction-field-correction factor the same values as in Ref. [34], the experimental estimate of $\Theta$ for $\mathrm{CSe}_{2}$ is revised to $3.47 \pm 0.60$ a.u., with a small but not negligible shift towards the calculated results.

\subsection{Assessment of the calculated Buckingham birefringences}

The last four columns of Tables 3 and 4 allow us to comment on the magnitude of the Buckingham birefringence in the $\mathrm{CX}_{2}(\mathrm{X}=\mathrm{O}, \mathrm{S}, \mathrm{Se}, \mathrm{Te})$ series. First, we observe that the temperature-independent contribution to ${ }_{\mathrm{m}} Q(\omega, T)$ in $\mathrm{CO}_{2}$ never exceeds $1 \%$ of the temperaturedependent contribution in column $F(\omega) / T$ of Tables 3 and 4. This percentage rises to $2 \%$ for $\mathrm{CSe}_{2}$ and $\mathrm{CTe}_{2}$ and to $3 \%$ for $\mathrm{CS}_{2}$. Still, the neglect of the temperature-independent contribution to the Buckingham birefringence in the $\mathrm{CX}_{2}(\mathrm{X}=\mathrm{O}, \mathrm{S}, \mathrm{Se}, \mathrm{Te})$ series is a good approximationfor example, we have seen that, when $b(\omega)$ is taken into account in the derivation of the quadrupole moment of $\mathrm{CSe}_{2}$, the quadrupole moment changes by only $1.2 \%$.

Because of the large experimental error bars, our calculated value for the Buckingham constant ${ }_{\mathrm{m}} Q(\omega, T)$ at $632.8 \mathrm{~nm}$ and $298.15 \mathrm{~K}$ is in good agreement with the experimental value (at $298 \mathrm{~K}$ ) for $\mathrm{CSe}_{2}$, in spite of our overestimation of the quadrupole moment $\Theta$ and the electric-dipole polarizability anisotropy $\Delta \alpha(-\omega ; \omega)$.

The discrepancies between theory and experiment for these quantities are also responsible for our underestimation of the magnitude of ${ }_{\mathrm{m}} Q(\omega, T)$ for $\mathrm{CO}_{2}$ and $\mathrm{CS}_{2}$, the $\mathrm{HF}$ values being closer to experiment than the DFT values. A reasonable value for the static electric field gradient in measurements of Buckingham birefringence is $\nabla E \approx$ $1 \times 10^{9} \mathrm{Vm}^{-2}$. With this value, we predict for the higher member of our series an anisotropy $\Delta n \approx 3 \times 10^{-13}$, which, for an optical path length of $1 \mathrm{~m}$, yields a retardation of $3 \times 10^{-6}$ rad at $632.8 \mathrm{~nm}$, well above the current limits of detection.

\section{Concluding remarks}

We have presented the first four-component relativistic study of Buckingham birefringence, using LAOs to ensure gauge-origin independence of the calculated results and, more importantly for the small systems considered here, to improve basis-set convergence. With the use of LAOs, the results obtained at the u-aug-DZ level of theory are within $5 \%$ of the estimated basis-set limit for the Buckingham birefringence of the molecules studied in this work. Electron correlation has been described using KS theory.

We have investigated the importance of relativity and electron correlation for the description of the Buckingham birefringence of the four nonpolar molecules $\mathrm{CO}_{2}, \mathrm{CS}_{2}, \mathrm{CSe}_{2}$, and $\mathrm{CTe}_{2}$. Electron correlation is significant, leading to changes of $10-15 \%$ relative to the DC HF results. However, the ability of the standard XC functionals LDA, BLYP, B3LYP, PBE and PBE0 investigated here to recover the correlation contribution to Buckingham birefringence is doubtful, noting that the agreement with earlier CCSD values is good for $\mathrm{CO}_{2}$ but poor for $\mathrm{CS}_{2}$, for which only one third of the electron-correlation effects are recovered. Further studies on the adequacy of modern XC functionals in describing electron-correlation contributions to Buckingham birefringence appear necessary.

In contrast to electron correlation, the effects of relativity on the temperature-independent contribution to Buckingham birefringence are negligible. The only exception to this observation in the series is $\mathrm{CTe}_{2}$, where the relativistic corrections amount to $20-30 \%$. The importance of relativity for this molecule is due to lowlying, resonant states becoming dipole allowed when spinorbit interactions are included in the calculations.

Relativistic effects have been found to be more important for the temperature-dependent contribution to Buckingham birefringence, where the quadrupole moments display fairly large relativistic corrections considering that the property probes the outer regions of the electron density. These relativistic corrections are almost exclusively scalar in nature, and are found to be due to the relativistic contraction of $\sigma$ valence orbitals. It would therefore be of interest to also investigate relativistic effects on polar 


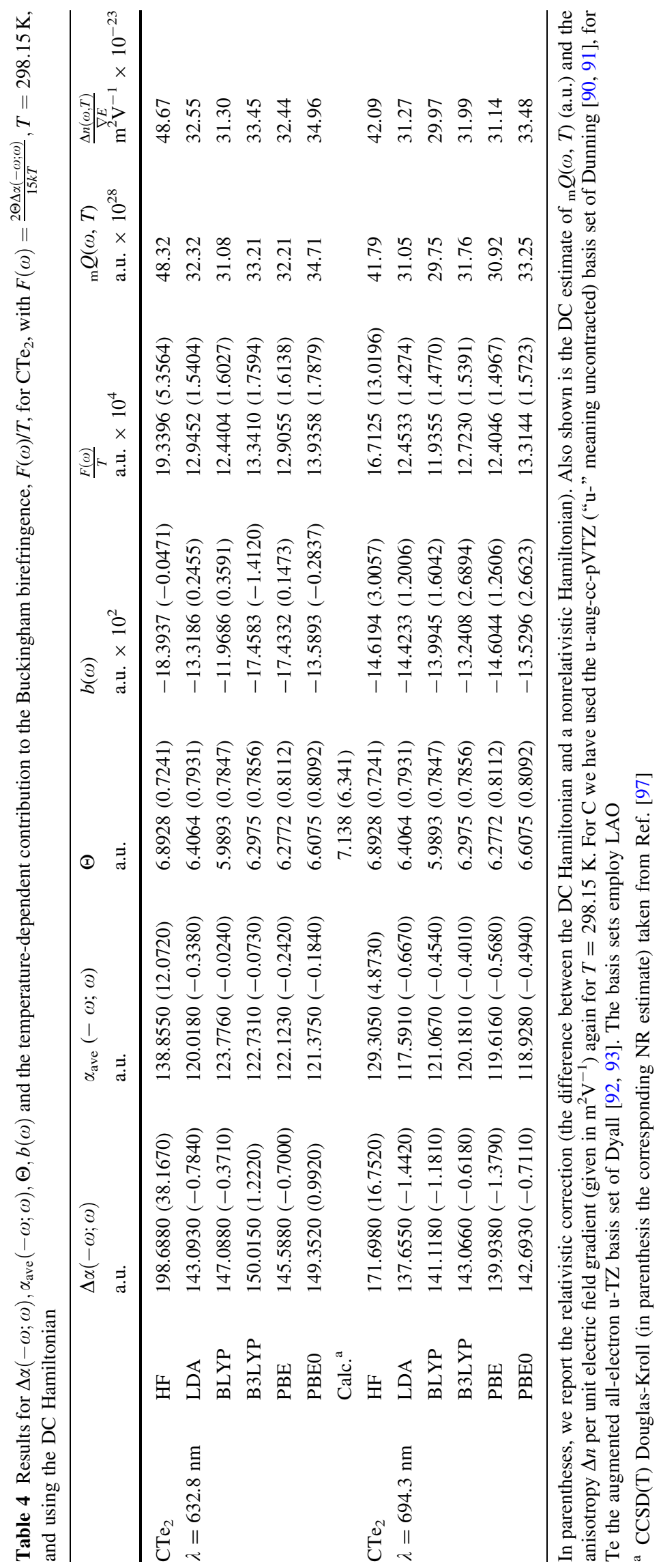


molecules, as additional contributions to the temperaturedependent part enters the expression for the Buckingham birefringence in this case.

Acknowledgments It is a pleasure for us to dedicate this work to Prof. Pekka Pyykkö, a pioneer in the development and understanding of relativistic effects in molecular properties. The authors thank Miroslav Iliaš, Małgorzata Olejniczak, Trond Saue and Andreas J. Thorvaldsen for helpful discussions. This work has received support from the Research Council of Norway through a Centre of Excellence Grant (Grant No. 179568/V30) and Grant No. 191251/V30. A grant of computer time from the Programme for Supercomputing is also gratefully acknowledged.

Open Access This article is distributed under the terms of the Creative Commons Attribution Noncommercial License which permits any noncommercial use, distribution, and reproduction in any medium, provided the original author(s) and source are credited.

\section{References}

1. Buckingham AD (1959) J Chem Phys 30:1580

2. Buckingham AD (1998) Annu Rev Phys Chem 49:XIII

3. Buckingham AD, Disch RL (1963) Proc R Soc Lond Ser A 273:275

4. Buckingham AD, Longuet-Higgins HC (1968) Mol Phys 14:63

5. Buckingham AD, Disch RL, Dunmur DA (1968) J Am Chem Soc 90:3104

6. Buckingham AD, Jamieson MJ (1971) Mol Phys 22:117

7. Ritchie GLD (1997) In: Clary DC, Orr B (eds) Optical electric and magnetic properties of molecules. Elsevier, Amsterdam

8. Coriani S, Halkier A, Rizzo A (2001) In: Pandalai G (eds) Recent research developments in chemical physics, vol 2. Transworld Scientific, Kerala, pp 1-21

9. Rizzo A, Coriani S (2005) Adv Quantum Chem 50:143. doi: 10.1016/S0065-3276(05)50008-X

10. Christiansen O, Coriani S, Gauss J, Hättig C, Jørgensen P, Pawłowski F, Rizzo A (2006) In: Papadopoulos MG, Sadlej AJ, Leszczynski J (eds) Non-linear optical properties of matter: from molecules to condensed phases, challenges and advances in computational chemistry and physics, vol 1. Springer, pp 51-99. ISBN: 1-4020-4849-1

11. Rizzo A (2007) In: Mennucci B, Cammi R (eds) Continuum solvation methods in chemical physics: theory and application. Wiley, pp 252-264. ISBN: 978-0-470-02938-1

12. Coriani S, Hättig C, Jørgensen P, Rizzo A, Ruud K (1998) J Chem Phys 109:7176

13. Halkier A, Coriani S, Jørgensen P (1998) Chem Phys Lett 294:292

14. Rizzo A, Coriani S, Halkier A, Hättig C (2000) J Chem Phys 113:3077

15. Coriani S, Halkier A, Jonsson D, Gauss J, Rizzo A, Christiansen O (2003) J Chem Phys 118:7329. doi:10.1063/1.1562198

16. Cappelli C, Ekström U, Rizzo A, Coriani S (2004) J Comp Meth Sci Eng (JCMSE) 4:365

17. Coriani S, Halkier A, Rizzo A, Ruud K (2000) Chem Phys Lett 326:269

18. Rizzo A, Cappelli C, Jansik B, Jonsson D, Salek P, Coriani S, Ågren H (2004) J Chem Phys 121:8814. doi:10.1063/1.1802771. Erratum ibid., 129, 039901 (2008)

19. Rizzo A, Cappelli C, Jansik B, Jonsson D, Salek P, Coriani S, Wilson DJD, Helgaker T, Ågren H (2005) J Chem Phys
122:234314. doi:10.1063/1.1935513. Erratum ibid., 129, 039901 (2008)

20. Rizzo A, Cappelli C, Junquera-Hernández JM, Sánchez de Merás AMJ, Sánchez-Marín J, Wilson DJD, Helgaker T (2005) J Chem Phys 123:114307. doi:10.1063/1.2034487. Erratum ibid., 129, 039901 (2008)

21. Buckingham AD, Coriani S, Rizzo A (2007) Theor Chem Acc 117:969. doi:10.1007/s00214-006-0217-y. Erratum ibid., 117, 979 (2007)

22. Shcherbin D, Thorvaldsen AJ, Ruud K, Coriani S, Rizzo A (2009) Phys Chem Chem Phys 11:816. doi:10.1039/b815752a

23. Rizzo A, Frediani L, Ruud K (2007) J Chem Phys 127:164321. doi:10.1063/1.2787527

24. Imrie DA, Raab RE (1991) Mol Phys 74:833

25. Raab RE, De Lange OL (2005) Multipole theory in electromagnetism. Classical, quantum and symmetry aspects, with applications. International Series of Monographs in Physics, 128. Oxford Science Publications, Clarendon Press, Oxford

26. Raab RE, De Lange OL (2003) Mol Phys 101:3467. doi:10.1080/ 00268970310001644612

27. De Lange OL, Raab RE (2004) Mol Phys 102:125. doi:10.1081/ 00268970410001668589

28. Marchesan D, Coriani S, Rizzo A (2003) Mol Phys 101:1851. doi:10.1080/0026897031000108122

29. Battaglia MR, Buckingham AD, Neumark D, Pierens RK, Williams JH (1981) Mol Phys 43:1015

30. Watson JN, Craven IE, Ritchie GLD (1997) Chem Phys Lett 274:1

31. Graham C, Pierrus J, Raab RE (1989) Mol Phys 67:939

32. Ritchie GLD, Vrbancich J (1980) J Chem Soc Farad T 2 76:1245

33. Graham C, Imrie DA, Raab RE (1998) Mol Phys 93:49

34. Brereton MP, Cooper MK, Dennis GR, Ritchie GLD (1981) Aust J Chem 34:2253

35. Bast R, Thorvaldsen AJ, Ringholm M, Ruud K (2009) Chem Phys 356:177. doi:10.1016/j.chemphys.2008.10.033

36. London F (1937) J Phys Paris 8:397

37. Ditchfield R (1972) J Chem Phys 56:5688

38. Hameka HF (1958) Mol Phys 1:203

39. Wolinski K, Hinton JF, Pulay P (1990) J Am Chem Soc 112:8251

40. Helgaker T, Jørgensen P (1991) J Chem Phys 95:2595

41. Barron LD (1982) Molecular light scattering and optical activity. Cambridge University Press, Cambridge

42. Pauli W (1927) Z Phys 43:601

43. Kutzelnigg W (1989) Z Phys D Atom Mol Cl 11:15

44. Manninen P, Lantto P, Vaara J, Ruud K (2003) J Chem Phys 119:2623. doi:10.1063/1.1586912

45. Visscher L, Saue T (2000) J Chem Phys 113:3996

46. Saue $T$ (2005) Adv Quantum Chem 48:383. doi:10.1016/ S0065-3276(05)48020-X

47. Pyykkö P (1988) Chem Rev 88:563

48. Dyall KG, Fægri K Jr. (2007) Introduction to relativistic quantum chemistry. Oxford University Press, Oxford

49. Gaunt JA (1929) Proc R Soc Lond A 122:513

50. Breit G (1929) Phys Rev 34:0553

51. Itoh $T$ (1965) Rev Mod Phys 37:159

52. Saue T (1995) Ph.D. thesis, University of Oslo

53. Lévy-Leblond JM (1967) Commun Math Phys 6:286

54. Lévy-Leblond JM (1970) Ann Phys 57:481

55. Saue T, Visscher L (2003) In: Wilson S, Kaldor U (eds) Theoretical chemistry and physics of heavy and superheavy elements. Kluwer, Dordrecht

56. Helgaker T, Hennum AC, Klopper W (2006) J Chem Phys 125:024102. doi:10.1063/1.2198527

57. Stanton RE, Havriliak S (1984) J Chem Phys 81:1910

58. Komorovský S, Repiský M, Malkina OL, Malkin VG, Malkin Ondík I, Kaupp M (2008) J Chem Phys 128:104101 
59. Cheng L, Xiao Y, Liu W (2009) J Chem Phys 131:244113

60. Thorvaldsen AJ, Ruud K, Kristensen K, Jørgensen P, Coriani S (2008) J Chem Phys 129:214108. doi:10.1063/1.2996351

61. Bast R, Ekström U, Gao B, Helgaker T, Ruud K, Thorvaldsen AJ (2011) Phys Chem Chem Phys 13:2627. doi:10.1039/C0 CP01647K

62. Krykunov M, Autschbach J (2005) J Chem Phys 123:114103. doi: $10.1063 / 1.2032428$

63. Casida ME (1995) In: Chong DP (ed) Recent advances in desity functional methods, vol I. World Scientific, Singapore

64. Bauernschmitt R, Ahlrichs R (1996) Chem Phys Lett 256:454

65. Rizzo A, Helgaker T, Ruud K, Barszczewicz A, Jaszunski M, Jørgensen P (1995) J Chem Phys 102:8953

66. Gao B, Thorvaldsen AJ, Ruud K (2011) Int J Quantum Chem 111:858

67. Salek P, Vahtras O, Helgaker T, Ågren H (2002) J Chem Phys 117:9630. doi:10.1063/1.1516805

68. Krykunov M, Banerjee A, Ziegler T, Autschbach J (2005) J Chem Phys 122:074105. doi:10.1063/1.1850919

69. Kjærgaard T, Jørgensen P, Thorvaldsen AJ, Salek P, Coriani S (2009) J Chem Theory Comput 5:1997. doi:10.1021/ct9001625

70. Bast R, Jensen HJ Aa, Saue T (2009) Int J Quantum Chem 109:2091. doi:10.1002/qua.22065

71. Ekström U, Visscher L, Bast R, Thorvaldsen AJ, Ruud K (2010) J Chem Theory Comput 6:1971. doi:10.1021/ct100117s

72. Saue T, Jensen HJ Aa (2003) J Chem Phys 118:522. doi:10.1063/ 1.1522407

73. Saue T, Jensen HJ Aa (1999) J Chem Phys 111:6211

74. Development version of DIRAC, a relativistic ab initio electronic structure program, Release DIRAC10 (2010), written by Saue T, Visscher L, Jensen HJ Aa, with contributions from Bast R, Dyall KG, Ekström U, Eliav E, Enevoldsen T, Fleig T, Gomes ASP, Henriksson J, Iliaš M, Jacob Ch R, Knecht S, Nataraj HS, Norman P, Olsen J, Pernpointner M, Ruud K, Schimmelpfennig B, Sikkema J, Thorvaldsen A, Thyssen J, Villaume S, Yamamoto S (see http://dirac.chem.vu.nl)

75. Slater JC (1951) Phys Rev 81:385

76. Vosko SH, Wilk L, Nusair M (1980) Can J Phys 58:1200

77. Becke AD (1988) Phys Rev A 38:3098

78. Lee CT, Yang WT, Parr RG (1988) Phys Rev B 37:785
79. Miehlich B, Savin A, Stoll H, Preuss H (1989) Chem Phys Lett 157:200

80. Stephens PJ, Devlin FJ, Chabalowski CF, Frisch MJ (1994) J Phys Chem US 98:11623

81. Becke AD (1993) J Chem Phys 98:5648

82. Perdew JP, Burke K, Ernzerhof M (1996) Phys Rev Lett 77:3865

83. Adamo C, Barone V (1999) J Chem Phys 110:6158

84. Ekström U (2010) Xcfun library. http://www.admol.org/xcfun

85. (1987) In: Landholt-Börnstein: structure data of free polyatomic molecules, vol II-15. Springer, pp 252-264

86. Maki AG, Sams RL (1981) J Mol Spectrosc 90:215

87. Frisch MJ, Trucks GW, Schlegel HB, Scuseria GE, Robb MA, Cheeseman JR, Scalmani G, Barone V, Mennucci B, Petersson GA, Nakatsuji H, Caricato M, Li X, Hratchian HP, Izmaylov AF, Bloino J, Zheng G, Sonnenberg JL, Hada M, Ehara M, Toyota K, Fukuda R, Hasegawa J, Ishida M, Nakajima T, Honda Y, Kitao O, Nakai H, Vreven T, Montgomery JA Jr, Peralta JE, Ogliaro F, Bearpark M, Heyd JJ, Brothers E, Kudin KN, Staroverov VN, Kobayashi R, Normand J, Raghavachari K, Rendell A, Burant JC, Iyengar SS, Tomasi J, Cossi M, Rega N, Millam JM, Klene M, Knox JE, Cross JB, Bakken V, Adamo C, Jaramillo J, Gomperts R, Stratmann RE, Yazyev O, Austin AJ, Cammi R, Pomelli C, Ochterski JW, Martin RL, Morokuma K, Zakrzewski VG, Voth GA, Salvador P, Dannenberg JJ, Dapprich S, Daniels AD, Farkas, Foresman JB, Ortiz JV, Cioslowski J, Fox DJ (2009) Gaussian 09 Revision A.1. Gaussian Inc., Wallingford

88. Weigend F, Ahlrichs R (2005) Phys Chem Chem Phys 7:3297

89. Peterson KA, Figgen D, Goll E, Stoll H, Dolg M (2003) J Chem Phys 119:11113. doi:10.1063/1.1622924

90. Dunning TH (1989) J Chem Phys 90:1007

91. Woon DE, Dunning TH (1994) J Chem Phys 100:2975

92. Dyall KG (1998) Theor Chem Acc 99:366. Addendum: Ibid. 108, 265 (2002)

93. Dyall KG (2006) Theor Chem Acc 115:441. doi:10.1007/s00214006-0126-0

94. Visscher L (1997) Theor Chem Acc 98:68

95. Visscher L, Dyall KG (1997) Atom Data Nucl Data 67:207

96. Bogaard MP, Buckingham AD, Pierens RK, White AH (1978) J Chem Soc Faraday Trans I 74:3008

97. Benkova Z, Sadlej AJ (2004) Mol Phys 102:687 